\title{
A prospective comparison of chronic primary angle- closure glaucoma versus primary open-angle glaucoma in Singapore
}

\author{
Cheryl SF Ngo ${ }^{1}$, Mmed, Maria Cecilia Aquino ${ }^{1}$, MD, Shabana Noor ${ }^{1}$, PhD, Seng Chee Loon ${ }^{1}$, FRCSE, \\ Chelvin CA $\underline{\text { Sng }}^{1}$, mMed, Gus Gazzard ${ }^{2}$, FRcophth, Wan-ling Wong ${ }^{3}$, BSc, Paul TK $\underline{\text { Chew }}^{1}$, FRCSE
}

\begin{abstract}
INTRODUCTION To describe the optic disc, visual field and ocular characteristics of a consecutive cohort of Asian patients with chronic primary angle-closure glaucoma (CPACG), and compare them with those having primary open-angle glaucoma (POAG).

METHODS In a prospective comparative case series of new patients with POAG or CPACG in Singapore, all patients underwent visual acuity assessment, slit-lamp examination, tonometry, gonioscopy, refraction, Heidelberg Retina Tomograph (HRT) and Humphrey visual field (HVF) assessment.

RESULTS 98 patients were enrolled (POAG $n=48$; CPACG $n=50)$. CPACG patients were significantly older $(66.5 \pm 9.2$ years vs. $64.1 \pm 13.5$ years; $p=0.027)$ and mostly female $(p=0.004)$. CPACG eyes had significantly higher intraocular pressure $(26.9 \pm 6.9 \mathrm{mmHg}$ vs. $24.5 \pm 3.3 \mathrm{mmHg} ; \mathrm{p}=0.03)$, shorter axial length $(22.89 \pm 0.97 \mathrm{~mm}$ vs. $24.26 \pm 1.79 \mathrm{~mm}$; $p<0.001)$ and shallower anterior chamber depth $(2.60 \pm 0.25 \mathrm{~mm}$ vs. $3.16 \pm 0.48 \mathrm{~mm} ; \mathrm{p}<0.001)$. HVF mean deviation or pattern standard deviation (PSD) did not differ significantly between POAG and CPACG eyes, but the latter had a lower PSD for a given mean deviation. HRT parameters between the two groups were not significantly different.

CONCLUSION In this study, CPACG eyes had significantly higher presenting intraocular pressure than POAG eyes, but there were no significant differences in optic disc topography. A majority of the patients in both groups had moderate field defects at the time of presentation, followed by severe and then mild defects. The field loss in CPACG eyes was more diffuse than that in POAG eyes.
\end{abstract}

Keywords: field of vision, intraocular pressure, optic nerve

\section{INTRODUCTION}

Primary angle-closure glaucoma (PACG) is a major health problem in Singapore. The rate of PACG in Asians is far higher than that in Caucasians, ${ }^{(1,2)}$ and it is thought that PACG is a more visually destructive disease compared to primary open-angle glaucoma (POAG). ${ }^{(3)}$ Several studies have documented the outcomes and response to treatment of an acute symptomatic attack of PACG, but few have adequately examined the disease characteristics of chronic primary angle-closure glaucoma (CPACG). ${ }^{(4-6)}$ Most previous studies on the patterns of visual field loss and optic disc changes in glaucoma have been conducted in patients with POAG. ${ }^{(7-9)}$ There is comparatively scarce data on the characteristics of field loss and ocular characteristics in patients with CPACG.

In this study, we selected an exclusive group of patients with CPACG who had no previous symptoms of acute attacks such as headache, nausea, vomiting and eye pain. To our knowledge, there are no studies comparing the disease characteristics or natural history of POAG and CPACG at the time of their first presentation to clinics. We also aimed to describe in detail the optic disc, visual field and ocular characteristics (such as intraocular pressure [IOP] at presentation, best-corrected visual acuity, sphere equivalent, mean keratometry, axial length, lens thickness, corneal thickness and lens opacity) of a consecutive cohort of Asian patients with CPACG, and to compare them with a similarly enrolled sample of patients with POAG.

\section{METHODS}

This was a prospective comparative study of new patients with POAG or CPACG, who presented to the National University Health System, a tertiary eye care centre in Singapore, from January 2002 to June 2009. These were newly diagnosed patients, with no previous treatment or laser therapy done. The patients, who presented with unrelated eye conditions, were found to have clinical signs suggestive of glaucoma. They were referred from general ophthalmology clinics and community screening programmes for diabetes mellitus and glaucoma, as they were found to have suspicious discs or raised IOP. Ethics approval was obtained from the institutional review board and the study was conducted in accordance with the Declaration of Helsinki. Written informed consent was obtained from all study participants prior to enrolment in the study.

CPACG was defined as visual field defect, glaucomatous optic neuropathy and at least one recorded IOP $>21 \mathrm{mmHg}$ (among three readings taken) in the presence of an occludable

${ }^{1}$ Department of Ophthalmology, National University Health System, Singapore, ${ }^{2}$ Moorfields Eye Hospital, London, UK, ${ }^{3}$ Singapore Eye Research Institute, Singapore National Eye Centre, Singapore

Correspondence: Dr Cheryl SF Ngo, Associate Consultant, Department of Ophthalmology, National University Hospital, 1E Kent Ridge Road, NUHS Tower Block, Level 7, Singapore 119228. cherylngo@gmail.com 
Table I. Demographic characteristics of patients with POAG or CPACG.

\begin{tabular}{|c|c|c|c|c|}
\hline \multirow[t]{2}{*}{ Characteristic } & \multicolumn{3}{|c|}{ No. of patients (\%) } & \multirow[t]{2}{*}{ p-value } \\
\hline & POAG (n = 48) & CPACG $(n=50)$ & All $(n=98)$ & \\
\hline Mean age \pm SD (yrs) & $64.1 \pm 13.5$ & $66.5 \pm 9.2$ & $65.3 \pm 11.5$ & $0.027^{*}$ \\
\hline $\begin{array}{c}\text { Ethnicity } \\
\text { Chinese } \\
\text { Malay } \\
\text { Indian } \\
\text { Other }\end{array}$ & $\begin{array}{c}35(72.9) \\
8(16.7) \\
5(10.4) \\
0(0.0)\end{array}$ & $\begin{array}{c}44(88.0) \\
5(10.0) \\
0(0.0) \\
1(2.0)\end{array}$ & $\begin{array}{c}79(80.6) \\
13(13.3) \\
5(5.1) \\
1(1.0)\end{array}$ & 0.05 \\
\hline $\begin{array}{l}\text { Gender } \\
\text { Male } \\
\text { Female }\end{array}$ & $\begin{array}{l}33(68.8) \\
15(31.2)\end{array}$ & $\begin{array}{l}20(40.0) \\
30(60.0)\end{array}$ & $\begin{array}{l}53(54.1) \\
45(45.9)\end{array}$ & $0.004^{*}$ \\
\hline Family history of glaucoma & $4(8.3)$ & $4(8.0)$ & $8(8.2)$ & 0.31 \\
\hline
\end{tabular}

${ }^{*} p<0.05$ was statistically significant for POAG vs. CPACG.

CPACG: chronic primary angle-closure glaucoma; POAG: primary open-angle glaucoma; SD: standard deviation

angle and peripheral anterior synechiae (PAS). An occludable angle was defined as one in which the posterior, usually pigmented, trabecular meshwork was not seen over 2,700 or more of the angle without indentation. ${ }^{(10,11)}$ Patients were asymptomatic at the time of presentation with no symptoms of acute attacks such as headache, nausea, vomiting and eye pain. Visual field defect consisted of either two points reduced by $>5 \mathrm{~dB}$ or one point reduced by $>10 \mathrm{~dB}$ below the age-specific threshold. ${ }^{(10,12)}$ Secondary causes for angle closure, including iris neovascularisation, lens intumescence, posterior segment mass, prior penetrating trauma and previous cataract or other ocular surgery, were excluded. Patients with serious medical conditions were also excluded from the study. POAG was defined as visual field defect, glaucomatous optic neuropathy and at least one recorded IOP $>21 \mathrm{mmHg}$ (among the three readings taken) in the presence of an open angle.

A complete ophthalmic examination, which consisted of visual acuity assessment, slit-lamp examination, tonometry, gonioscopy, refraction, scanning laser ophthalmoscopic optic nerve assessment and Humphrey visual field (HVF) assessment, was performed for each patient. Anterior chamber depth, lens thickness and axial length measurements were obtained using the IOLMaster (Carl Zeiss, Jena, Germany). Data were collected for only one eye of each patient in the study. If both eyes were glaucomatous, then one eye was randomly selected for statistical analysis.

The degree of lens opacity - nuclear opacity and colour, and cortical and posterior subcapsular opacities - was graded by clinical observation with a slit lamp using standard photographs of the Lens Opacities Classification System (LOCS) III scheme. Tonometry was performed using the Goldmann tonometer (HaagStreit, Mason, $\mathrm{OH}$, USA). Three readings were taken from each eye and the time of the readings was recorded. For analytical purposes, the median of these readings was taken as the IOP in that eye. The two-mirror gonioscopy lens was applied to the anaesthetised cornea, using hydroxypropyl methylcellulose (HPMC, $0.5 \%$ ) as a coupling agent. The appearance of the drainage angles in each quadrant was recorded and classified as open or narrow using the modified Scheie classification. ${ }^{(13)}$ Indentation with a Sussman four-mirror lens (Ocular Instruments,
Bellevue, WA, USA) was used to assess the presence and extent of PAS and pigment in the trabecular meshwork.

The optic nerve head of all eyes was imaged using the Heidelberg Retina Tomograph II (HRT II) version 1.5.0 (Heidelberg Engineering $\mathrm{GmbH}$, Heidelberg, Germany). A trained project technician acquired all the images. Each patient had three highquality scans, and the mean morphometric parameters derived from the three scans were used for analysis. The optic disc area, cup area, cup volume, cup-disc area ratio, rim area and rim volume were assessed.

The standard 24-2 testing algorithm of the HVF analyser (Carl Zeiss Meditec, Dublin, CA, USA) was used with the full threshold algorithm. Three tests were performed within two weeks, and the third test was used to minimise the known learning effects seen with the earlier tests. The tests were considered reliable and eligible for analysis if they were completed with less than $33 \%$ false positives, less than $33 \%$ false negatives and less than $20 \%$ fixation losses. Glaucomatous field defect was defined as two points reduced by $>5 \mathrm{~dB}$ or one point reduced by $>10 \mathrm{~dB}$ below the age-specific threshold. The Humphrey statistical software package (STATPAC; Carl Zeiss Meditec, Dublin, CA, USA) was used to analyse the results.

Data were analysed using the Statistical Package for the Social Sciences for Windows version 17.0 (SPSS Inc, Chicago, IL, USA). Demographic and ophthalmologic characteristics were compared using the $t$-test. A p-value $<0.05$ was considered to be statistically significant. Multiple regression models were generated to detect any differences in HRT II parameters between POAG and CPACG eyes. Optic disc characteristics at a particular level of damage, as determined by HVF pattern standard deviation (PSD), were compared between patients with POAG and those with CPACG. Disc area was also controlled for, as it has been reported to have an impact on other disc measures. ${ }^{(14)}$

\section{RESULTS}

A total of 98 patients were enrolled in the study (POAG $\mathrm{n}=48$; CPACG $\mathrm{n}=50$ ). Tables I and II present the demographic and ocular characteristics of patients with POAG and CPACG. The demographics of our patient groups represent that of the population of Singapore. Patients with CPACG were significantly 
Table II. Ocular characteristics of patients with POAG or CPACG.

\begin{tabular}{lcccc}
\hline Characteristic & \multicolumn{3}{c}{ Mean \pm SD } & p-value \\
\cline { 2 - 4 } & POAG $(\mathbf{n}=\mathbf{4 8})$ & CPACG $(\mathbf{n}=\mathbf{5 0})$ & All $(\mathbf{n}=\mathbf{9 8})$ & \\
\hline Intraocular pressure at presentation (mmHg) & $24.50 \pm 3.30$ & $26.90 \pm 6.90$ & $25.70 \pm 5.50$ & $0.03^{*}$ \\
Best-corrected visual acuity (logMAR) & $0.15 \pm 0.19$ & $0.17 \pm 0.16$ & $0.16 \pm 0.18$ & 0.46 \\
Sphere equivalent (dioptres) & $-1.80 \pm 3.51$ & $-0.05 \pm 2.06$ & $-0.91 \pm 3.00$ & $<0.001^{*}$ \\
Mean keratometry (dioptres) & $44.52 \pm 1.80$ & $44.43 \pm 1.55$ & $44.48 \pm 1.66$ & 0.80 \\
Axial length (mm) & $24.26 \pm 1.79$ & $22.89 \pm 0.97$ & $23.55 \pm 1.57$ & $<0.001^{*}$ \\
Anterior chamber depth (mm) & $3.16 \pm 0.48$ & $2.60 \pm 0.25$ & $2.86 \pm 0.47$ & $<0.001^{*}$ \\
Lens thickness (mm) & $4.42 \pm 0.51$ & $4.66 \pm 0.75$ & $4.55 \pm 0.66$ & 0.08 \\
Corneal thickness (mm) & $0.45 \pm 0.23$ & $0.57 \pm 0.40$ & $0.51 \pm 0.17$ & $<0.001^{*}$ \\
LOcs & & & & \\
$\quad$ Nuclear colour & $2.23 \pm 1.07$ & $2.20 \pm 0.93$ & $2.20 \pm 1.00$ & 0.86 \\
Nuclear opalescence & $2.23 \pm 1.09$ & $1.88 \pm 0.92$ & $2.00 \pm 1.00$ & 0.12 \\
Cortical opacity & $1.85 \pm 1.00$ & $2.22 \pm 0.95$ & $2.10 \pm 0.96$ & 0.16 \\
Posterior subcapsular & $1.64 \pm 0.73$ & $2.11 \pm 0.90$ & $2.00 \pm 0.90$ & $0.04^{*}$ \\
\hline
\end{tabular}

${ }^{*} p<0.05$ was statistically significant for POAG vs. CPACG.

CPACG: chronic primary angle-closure glaucoma; LOCS: Lens Opacities Classification System; logMAR: logarithm of the minimum angle of resolution; POAG: primary open-angle glaucoma; SD: standard deviation

Table III. Results of least square linear regression analysis for intraocular pressure.

\begin{tabular}{lcl}
\hline Variable & $\boldsymbol{\beta}(\mathbf{S E})$ & $\mathbf{p}$-value \\
\hline Gender & 0.099 & 0.361 \\
Age & -0.121 & 0.240 \\
Cornea thickness & -0.089 & 0.404 \\
Glaucoma type $^{\dagger}$ & 0.271 & $0.014^{*}$ \\
\hline
\end{tabular}

${ }^{\star} p<0.05$ was statistically significant for POAG vs. CPACG. ${ }^{\dagger} \beta$ coefficients represent the difference between dependent variables for POAG vs. CPACG.

SE: standard error

Table IV. Comparison of HVF parameters.

\begin{tabular}{|c|c|c|c|}
\hline & \multicolumn{2}{|c|}{ Mean \pm SD } & \multirow[t]{2}{*}{ p-value } \\
\hline & $\begin{array}{c}\text { POAG } \\
(n=48)\end{array}$ & $\begin{array}{l}\text { CPACG } \\
(n=50)\end{array}$ & \\
\hline HVF mean deviation $(\mathrm{dB})^{*}$ & $-8.81 \pm 9.00$ & $-9.07 \pm 8.16$ & 0.88 \\
\hline HVF PSD $(d B) *$ & $5.16 \pm 3.93$ & $5.02 \pm 3.32$ & 0.86 \\
\hline
\end{tabular}

older than those with POAG $(66.5 \pm 9.2$ years vs. $64.1 \pm$ 13.5 years; $p=0.027)$ and consisted of a higher proportion of women $(p=0.004)$. There were no significant differences between the two groups with regard to ethnicity $(p=0.05)$ or positive family history of glaucoma $(p=0.31)$. POAG eyes had significantly higher myopia compared to CPACG eyes $(-1.80 \pm$ 3.51 dioptres vs. $-0.05 \pm 2.06$ dioptres; $\mathrm{p}<0.001)$ and thinner corneas $(0.45 \pm 0.227 \mathrm{~mm}$ vs. $0.57 \pm 0.4 \mathrm{~mm} ; \mathrm{p}<0.001)$. CPACG eyes had a significantly higher IOP at presentation compared to POAG eyes $(26.9 \pm 6.9 \mathrm{mmHg}$ vs. $24.5 \pm 3.3 \mathrm{mmHg}$; $\mathrm{p}=0.03$ ) and a mean of 3,600 of PAS. The time at which IOP was taken was not significantly different between the two groups. CPACG eyes also had a shorter axial length compared to POAG eyes $(22.89 \pm 0.97 \mathrm{~mm}$ vs. $24.26 \pm 1.79 \mathrm{~mm} ; \mathrm{p}<0.001)$ and shallower anterior chamber depth $(2.60 \pm 0.25 \mathrm{~mm}$ vs.
$3.16 \pm 0.48 \mathrm{~mm} ; \mathrm{p}<0.001)$. There were no significant differences between the two groups in best-corrected visual acuity, mean keratometry, lens thickness and three out of the four LOCS III grades of lens opacity (only posterior subcapsular opacity had a significant difference $[p=0.04]$ )

Table III presents the results of the linear regression analysis for IOP between POAG and CPACG eyes. The difference in IOP between POAG and CPACG eyes was statistically significant even after adjustment for age, gender and corneal thickness. There was no significant difference in the HVF mean deviation $(-8.81 \pm 9.00 \mathrm{~dB}$ vs. $-9.07 \pm 8.16 \mathrm{~dB} ; \mathrm{p}=0.88)$ or HVF PSD $(5.16 \pm 3.93 \mathrm{~dB}$ vs. $5.02 \pm 3.32 \mathrm{~dB} ; \mathrm{p}=0.86)$ between POAG and CPACG eyes (Table IV). To further examine the differences in the pattern of field loss between POAG and CPACG eyes, the visual fields in these patients were quantified as mild, moderate or severe, based on the Advance Glaucoma Intervention Study (AGIS) score ${ }^{(15)}$ (Table V). The percentage of eyes with mild field defects $(12.2 \%$ vs. $12.5 \%)$, moderate defects ( $65.3 \%$ vs. $64.6 \%)$ and severe defects $(22.4 \%$ vs. $22.9 \%$ ) were comparable among patients with POAG and CPACG. Even after breaking them into groups, there was no significant difference in the severity of field defects between patients with POAG and CPACG based on AGIS scores. HRT II parameters such as rim area, rim volume and vertical cup-disc ratio were also not significantly different between patients with POAG and CPACG.

Table VI presents the results of the multiple linear regression analysis for HRT II parameters. Parameters were adjusted for HVF PSD to compare HRT II parameters at the same stage of disease. PSD values were used rather than mean deviation, as it would take away possible confounders (such as cataract) that could affect the mean deviation. After adjusting for HVF PSD and disc area, HRT II parameters were still not significantly different between patients with POAG and CPACG. Using Bonferroni correction, the $p$-value needed for significance was ascertained to be 0.0125 . Fig. 1 shows the relationship between mean 
Table V. Comparison of the results for HVF and HRT II between patients with POAG and CPACG having different grades of severity of field defects on Advanced Glaucoma Intervention Study score.

\begin{tabular}{|c|c|c|c|c|c|c|c|c|c|}
\hline \multirow[t]{2}{*}{ Variable } & \multicolumn{3}{|c|}{ Mild field defect } & \multicolumn{3}{|c|}{ Moderate field defect } & \multicolumn{3}{|c|}{ Severe field defect } \\
\hline & POAG & CPACG & p-value & POAG & CPACG & p-value & POAG & CPACG & p-value \\
\hline \multicolumn{10}{|l|}{ HVF (dB)* } \\
\hline PSD & $1.89 \pm 0.39$ & $1.83 \pm 0.53$ & 0.81 & $4.71 \pm 0.77$ & $4.28 \pm 2.44$ & 0.58 & $8.17 \pm 3.59$ & $8.94 \pm 3.07$ & 0.60 \\
\hline \multicolumn{10}{|l|}{ HRT II } \\
\hline Disc area* $\left(\mathrm{mm}^{2}\right)$ & $1.82 \pm 0.60$ & $2.01 \pm 0.22$ & 0.39 & $2.26 \pm 0.60$ & $2.18 \pm 0.53$ & 0.56 & $2.49 \pm 0.56$ & $2.41 \pm 0.63$ & 0.79 \\
\hline Rim volume $\left(\mathrm{mm}^{3}\right)$ & $0.25 \pm 0.07$ & $0.43 \pm 0.18$ & 0.14 & $0.30 \pm 0.14$ & $0.30 \pm 0.14$ & 0.73 & $0.21 \pm 0.21$ & $0.30 \pm 0.21$ & 0.39 \\
\hline Vertical cup-disc ratio* & $0.65 \pm 0.09$ & $0.45 \pm 0.19$ & 0.16 & $0.63 \pm 0.18$ & $0.73 \pm 0.81$ & 0.56 & $0.78 \pm 0.14$ & $0.69 \pm 0.22$ & 0.17 \\
\hline
\end{tabular}

Data is presented as mean \pm standard deviation. *Each parameter has been adjusted for age.

CPACG: chronic primary angle-closure glaucoma; HRT II: Heidelberg Retina Tomograph II; HVF: Humphrey visual field; POAG: primary open-angle glaucoma; PSD: pattern standard deviation.

Table VI. Results of multiple linear regression models for HRT II parameters.

\begin{tabular}{|c|c|c|c|c|c|c|}
\hline \multirow[t]{2}{*}{ Variable } & \multicolumn{2}{|c|}{ Vertical cup-disc ratio* } & \multicolumn{2}{|c|}{ Rim area* } & \multicolumn{2}{|c|}{ Rim volume* } \\
\hline & $\beta(\mathrm{SE})$ & p-value & $\beta$ (SE) & p-value & $\beta$ (SE) & p-value \\
\hline Glaucoma type $^{+}$ & $-0.02(0.10)$ & 0.793 & $-0.17(0.09)$ & 0.083 & $-0.04(0.03)$ & 0.195 \\
\hline HVF PSD & $0.000(0.01)$ & 0.951 & $-0.03(0.01)$ & 0.04 & $-0.005(0.005)$ & 0.274 \\
\hline HRT II disc area & $0.11(0.09)$ & 0.238 & $0.22(0.09)$ & 0.01 & $0.005(0.03)$ & 0.874 \\
\hline
\end{tabular}

Note: HRT II parameters were the dependent variable.

${ }^{*}$ Each model was adjusted for age, pattern standard deviation and disc area using Bonferroni correction; $p$-value needed for significance was set at 0.0125.

${ }^{\dagger} \beta$ coefficients represent the difference between dependent variables for POAG vs. CPACG.

CPACG: chronic primary angle-closure glaucoma; HRT II: Heidelberg Retina Tomograph II; HVF: Humphrey visual field; POAG: primary open-angle glaucoma; PSD: pattern standard deviation; SE: standard error

deviation and PSD in POAG and CPACG eyes. Although the relationship of PSD to mean deviation was similar for POAG and CPACG eyes, PSD was higher for POAG eyes except at the extremes of mean deviation. The best-fit quadratic curves for both groups showed that POAG eyes had higher PSD values for any given mean deviation.

\section{DISCUSSION}

A majority of patients with PACG are asymptomatic, with only a minority presenting with acute, symptomatic, high IOP. ${ }^{(16)}$ Therefore, in this prospective study, we chose to study patients with CPACG who were asymptomatic and compare them to a group of patients with POAG. We found that patients with CPACG had significantly higher IOP at the time of presentation than those with POAG. This finding is in contrast to a study by Boland et al, ${ }^{(17)}$ who found no significant difference between the PACG and POAG groups. This discrepancy in our findings may be attributed to the following reasons: (a) Boland et al's cohort of patients were patients recruited from a glaucoma clinic and many patients were either on glaucoma eye drops or had already undergone laser or glaucoma surgery; (b) racial differences; there were few Asians included in Boland et al's study, while our study consisted of solely Asian patients; (c) there were three times more patients with POAG compared to patients with PACG in Boland et al's study, while the number of patients with POAG and CPACG in our study were comparable; and (d) patients with both acute and chronic angle-closure, some of whom had even received treatments, were included in Boland et al's study, while our study included only patients with chronic angle-closure who had not received any treatment.

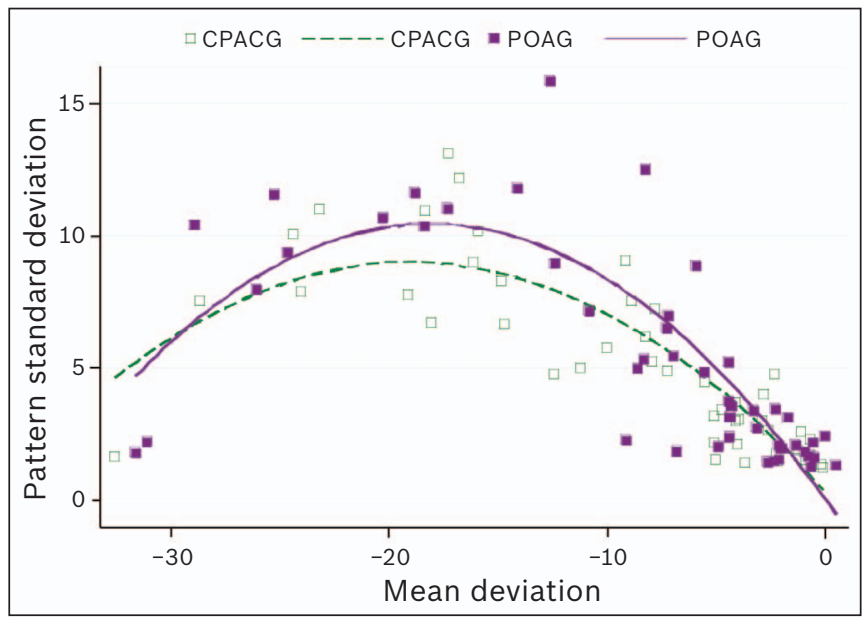

Fig. 1 Scattergram of pattern standard deviation and mean deviation for eyes with CPACG and POAG. Lines are best-fit quadratic functions for each group.

Thomas et al reported higher IOP in patients with PACG than in those with POAG, although the difference was not significant. ${ }^{(18)}$ There are several possible reasons for the markedly high IOP observed among our patients with CPACG. The natural history of primary angle-closure may be different in Asian eyes compared to that in patients of other races. Asian eyes have a genetic and anatomic predisposition to the development of angle-closure. ${ }^{(19-21)}$ Angle crowding by progressive thickening of the lens or a plateau iris configuration, together with shallow anterior chamber, as seen in our study, may play a role in the formation of PAS in Asian eyes, causing obstruction to aqueous outflow, resulting in high IOP and development of chronic angle-closure glaucoma. ${ }^{(19,21,22)}$ 
It is well known that central corneal thickness (CCT) can affect IOP measurements by tonometry. ${ }^{(23-27)}$ Thicker central cornea is associated with higher measured IOP. ${ }^{(28)}$ In our study, CCT was significantly different between CPACG and POAG eyes $(0.57 \pm 0.4 \mathrm{~mm}$ vs. $0.45 \pm 0.227 \mathrm{~mm} ; \mathrm{p}<0.001)$. Hence, the effects of CCT, as well as age and gender, on IOP measurements were taken into consideration. We found that after correcting for CCT, age and gender, IOP was still significantly higher in CPACG eyes than in POAG eyes in our study.

The overall recruitment for our study was representative of the Singapore population. We found that patients with CPACG were significantly older than patients with POAG, and there was a higher proportion of women with CPACG. Previous studies have found that women are more likely to develop angle-closure glaucoma. ${ }^{(21,29,30)}$ CPACG eyes also have significantly shorter axial lengths, shallower anterior chamber depths and less myopia than POAG eyes.

It would be expected that a higher IOP would result in more structural and functional damage of the optic nerve. ${ }^{(31,32)}$ However, when we compared the optic nerve head characteristics between patients with POAG and CPACG, who had different grades of severity of visual field defects based on $\mathrm{AGIS}^{(15)}$ scoring, there was no significant difference between the two groups of patients. Thomas et al, who also found no significant differences in HRT parameters between patients with PACG and POAG, reported that the mean disc area was larger in patients with PACG than in those with POAG in an Indian population. ${ }^{(18)}$ This is in contrast to our findings that patients with POAG had a slightly larger disc area than those with CPACG. It has been reported that eyes with high myopia ${ }^{(33,34)}$ and eyes with longer axial lengths ${ }^{(35)}$ are strongly correlated with larger optic discs. Indeed, in our study, POAG eyes had significantly higher myopic refractive error and longer axial lengths than CPACG eyes, which explains the larger discs observed among our patients with POAG.

Although not statistically significant, we also found that patients with CPACG had larger optic disc rim area and rim volume across all three grades of severity of disease than patients with POAG. Sihota et al have previously found that CPACG eyes have a larger rim area, as measured using optical coherence tomography, when compared to POAG eyes. The authors suggested that the moderately preserved neuroretinal rim area in CPACG eyes, albeit with comparable visual field loss, could be because glaucomatous damage in CPACG is partly due to intermittent rises in IOP whereas glaucomatous damage in POAG eyes is due to prolonged pressure. ${ }^{(36)}$ Despite the higher IOP in CPACG eyes, we found that HVF mean deviation and PSD did not differ significantly between POAG and CPACG eyes. There was also no significant difference in the severity of field loss between POAG and CPACG eyes when scored using the AGIS system. This suggests that the severity of visual field changes may not be due to IOP alone and that other factors, including the duration of insult, may be responsible for the severity of field loss in these patients.
Conversely, we did find a difference in the pattern of field loss among patients with POAG and CPACG. CPACG eyes had lower PSD values for a given mean deviation compared to POAG eyes in our study. This finding is similar to that of Boland et $\mathrm{al}^{(17)}$ suggesting that field loss in CPACG eyes is more diffuse than in POAG eyes. It has also been reported that there is generalised, rather than localised, field loss after an acute symptomatic attack of angle-closure. ${ }^{(37)}$ Interestingly, although our patients had CPACG, we found a pattern of generalised field loss similar to that seen in patients with acute attacks. Retrospective studies by Gazzard et $\mathrm{al}^{(10)}$ and Rhee et al ${ }^{(38)}$ have also found that patients with PACG had higher IOP and less focal field loss than those with POAG. Different patterns of field loss suggest that there are differences in the underlying pathophysiology - a more diffuse pattern of field loss in CPACG eyes may result from exposure to higher IOP, while localised field defects in POAG eyes may suggest non-pressure-related mechanisms of nerve damage. It is also possible that these patterns of field loss may be partly due to differences in IOP spikes, as fluctuations in IOP may be greater in CPACG eyes than POAG eyes, ${ }^{(39)}$ and this may be an independent risk factor for field loss. ${ }^{(40)}$

One limitation of our study is that it was hospital-based rather than population-based. However, we were able to capture a large group of patients with CPACG that was comparable in number to enrolled patients with POAG. A follow-up study would help to further characterise and compare the progression of these two diseases.

The incidence of POAG and CPACG is likely to rise with the ageing of the Singaporean population. ${ }^{(41)}$ Our study shows that patients with CPACG were asymptomatic at the time of their first presentation to the clinic and had significantly higher IOP than patients with POAG. CPACG eyes had lower PSD at the same level of mean deviation on HVF compared to POAG eyes, suggesting that field loss in CPACG eyes is more diffuse than in POAG eyes. A majority of patients in both groups had moderate field defects at the time of presentation, followed by severe and finally mild defects. There was no significant difference in the optic disc topographies of patients from the two groups. Our findings provide insight into the natural history of the disease in patients with POAG and CPACG. Our results also underscore the significance of community-based health education and prevention programmes targeting patients with such visually destructive diseases. Also, such programmes need to target subgroups of the population that are at the highest risk for these diseases.

\section{ACKNOWLEDGEMENTS}

We would like to acknowledge the following people, without whom the completion of this project would not have been possible: Jovina LS See, Alejandro Chung, Joseph Manuel Cruz, Grace Dizon, Marion Bayana, Narakorn Vimolchalao and Ma. Catherina Coronel. 


\section{REFERENCES}

1. Foster PJ, Oen FT, Machin D, et al. The prevalence of glaucoma in Chinese residents of Singapore: a cross-sectional population survey of the Tanjong Pagar district. Arch Ophthalmol 2000; 118:1105-11.

2. Tielsch JM, Sommer A, Katz J, et al. Racial variations in the prevalence of primary open-angle glaucoma. The Baltimore Eye Survey. JAMA 1991; 17:369-74.

3. Foster PJ. The epidemiology of primary angle closure and associated glaucomatous optic neuropathy. Semin Ophthalmol 2002; 17:50-8.

4. Aung T, Lim MC, Chan YH, Rojanapongpun P, Chew PT. Configuration of the drainage angle, intraocular pressure, and optic disc cupping in subjects with chronic angle-closure glaucoma. Ophthalmology 2005; 112:28-32.

5. Foulds WS, Phillips Cl. Some observations on chronic closed-angle glaucoma. Br J Ophthalmol 1957; 41:208-13.

6. Lau LI, Liu CJ, Chou JC, Hsu WM, Liu JH. Patterns of visual field defects in chronic angle-closure glaucoma with different disease severity. Ophthalmology 2003; 110:1890-4.

7. Goldberg I. Optic disc and visual field changes in primary open angle glaucoma. Aust J Ophthalmol 1981; 9:223-9.

8. Bartz-Schmidt KU, Thumann G, Jonescu-Cuypers CP, Krieglstein GK. Quantitative morphologic and functional evaluation of the optic nerve head in chronic open-angle glaucoma. Surv Ophthalmol 1999; 44 suppl 1:S41-53.

9. Macri A, Rolando M, Corallo G, et al. A comparative study of computerised visual field testing and optic disc morphometric parameters in the follow-up of primary open angle glaucoma. Eye (Lond) 1998; 12:916-20.

10. Gazzard G, Foster PJ, Viswanathan AC, et al. The severity and spatial distribution of visual field defects in primary glaucoma: a comparison of primary open-angle glaucoma and primary angle-closure glaucoma. Arch Ophthalmol 2002; 120:1636-43.

11. Foster PJ, Aung T, Nolan WP, et al. Defining "occludable" angles in population surveys: drainage angle width, peripheral anterior synechiae, and glaucomatous optic neuropathy in east Asian people. Br J Ophthalmol 2004; 88:486-90.

12. Saw SM, Gazzard G, Au Eong KG, Oen F, Seah S. Utility values in Singapore Chinese adults with primary open-angle and primary angle-closure glaucoma. J Glaucoma 2005; 14:455-62.

13. Scheie H. Width and pigmentation of the angle of the anterior chamber; a system of grading by gonioscopy. AMA Arch Ophthalmol 1957; 58:510-12

14. Bowd C, Zangwill LM, Blumenthal EZ, et al. Imaging of the optic disc and retinal nerve fiber layer: the effects of age, optic disc area, refractive error, and gender. J Opt Soc Am A Opt Image Sci Vis 2002; 19:197-207.

15. Advanced Glaucoma Intervention Study. 2. Visual field test scoring and reliability. Ophthalmology 1994; 101:1445-55

16. Sihota R. An Indian perspective on primary angle closure and glaucoma. Indian J Ophthalmol 2011; 59 Suppl:S76-81.

17. Boland MV, Zhang L, Broman AT, Jampel HD, Quigley HA. Comparison of optic nerve head topography and visual field in eyes with open-angle and angle-closure glaucoma. Ophthalmology 2008; 115:239-45.

18. Thomas R, Muliyil J, Simha RA, Parikh RS. Heidelberg Retinal Tomograph (HRT 2) parameters in primary open angle glaucoma and primary angle closure glaucoma: a comparative study in an Indian population. Ophthalmic Epidemiol 2006; 13:343-50.

19. Shabana N, Aquino MC, See J, et al. Quantitative evaluation of anterior chamber parameters using anterior segment optical coherence tomography in primary angle closure mechanisms. Clin Experiment Ophthalmol 2012; 40:792-80.

20. Friedman DS, Foster PJ, Aung T, He M. Angle closure and angle-closure glaucoma: what we are doing now and what we will be doing in the future. Clin Experiment Ophthalmol 2012; 40:381-7.

21. Amerasinghe N, Aung T. Angle-closure: risk factors, diagnosis and treatment. Prog Brain Res 2008; 173:31-45.

22. Wang N, Wu H, Fan Z. Primary angle closure glaucoma in Chinese and Western populations. Chin Med J (Engl) 2002; 115:1706-15.

23. Doughty MJ, Zaman ML. Human corneal thickness and its impact on intraocular pressure measures: a review and meta-analysis approach. Surv Ophthalmol 2000; 44:367-408.

24. Broman AT, Congdon NG, Bandeen-Roche K, Quigley HA. Influence of corneal structure, corneal responsiveness, and other ocular parameters on tonometric measurement of intraocular pressure. J Glaucoma 2007; 16:581-8.

25. Ehlers N. On corneal thickness and intraocular pressure. II. A clinical study on the thickness of the corneal stroma in glaucomatous eyes. Acta Ophthalmol (Copenh) 1970; 48:1107-12.

26. lester M, Mete M, Figus M, Frezzotti P. Incorporating corneal pachymetry into the management of glaucoma. J Cataract Refract Surg 2009; 35:1623-8.

27. Goldmann H, Schmidt T. [Applanation tonometry]. Ophthalmologica 1957; 134:221-42. German.

28. Wong TT, Wong TY, Foster PJ, et al. The relationship of intraocular pressure with age, systolic blood pressure, and central corneal thickness in an asian population. Invest Ophthalmol Vis Sci 2009; 50:4097-102.

29. Casson RJ, Baker M, Edussuriya K, et al. Prevalence and determinants of angle closure in central Sri Lanka: the Kandy Eye Study. Ophthalmology 2009; 116:1444-9.

30. Vijaya L, George R, Arvind H, et al. Prevalence of primary angle-closure disease in an urban south Indian population and comparison with a rural population. The Chennai Glaucoma Study. Ophthalmology 2008; 115:655-60.

31. Goldberg I. Relationship between intraocular pressure and preservation of visual field in glaucoma. Surv Ophthalmol 2003; 48 suppl 1:S3-7.

32. Morrison JC, Johnson EC, Cepurna W, Jia L. Understanding mechanisms of pressure-induced optic nerve damage. Prog Retin Eye Res 2005; 24:217-40.

33. Agarwal HC, Gulati V, Sihota R. The normal optic nerve head on Heidelberg Retina Tomograph II. Indian J Ophthalmol 2003; 51:25-33.

34. Jonas JB, Fernández MC, Naumann GO. Correlation of the optic disc size to glaucoma susceptibility. Ophthalmology 1991; 98:675-80.

35. Oliveira C, Harizman N, Girkin CA, et al. Axial length and optic disc size in normal eyes. Br J Ophthalmol 2007; 91:37-9.

36. Sihota R, Sony P, Gupta V, Dada T, Singh R. Comparing glaucomatous optic neuropathy in primary open angle and chronic primary angle closure glaucoma eyes by optical coherence tomography. Ophthalmic Physiol Opt 2005; 25:408-15.

37. Bonomi L, Marraffa M, Marchini G, Canali N. Perimetric defects after a single acute angle-closure glaucoma attack. Graefes Arch Clin Exp Ophthalmol 1999; 237:908-14.

38. Rhee K, Kim YY, Nam DH, Jung HR. Comparison of visual field defects between primary open-angle glaucoma and chronic primary angleclosure glaucoma in the early or moderate stage of the disease. Korean J Ophthalmol 2001; 15:27-31.

39. Shapiro A, Zauberman H. Diurnal changes of the intraocular pressure of patients with angle-closure glaucoma. Br J Ophthalmol 1979; 63:225-7.

40. Asrani S, Zeimer R, Wilensky J, et al. Large diurnal fluctuations in intraocular pressure are an independent risk factor in patients with glaucoma. J Glaucoma 2000; 9:134-42.

41. Statistics Singapore. Population in brief: 2009. In: Department of Statistics Singapore [online]. Available at: www.nptd.gov.sg/.../Population\%20in\%20 Brief\%202009.pdf. Accessed January 28, 2013. 